\title{
Seeing Streptococcus pneumoniae, a Common Killer Bacteria
}

\author{
Rikke Schmidt Kjærgaard \\ Aarhus University \\ Aarhus Institute of Advanced Studies, \\ \& Interdisciplinary Nanoscience Center, \\ Høegh-Guldbergs Gade 6B, DK-8000 Aarhus C, \\ Denmark \\ risk@aias.au.dk
}

\author{
Ebbe Sloth Andersen \\ Aarhus University \\ Interdisciplinary Nanoscience Center, \\ Gustav Wieds Vej 14, \\ DK-8000 Aarhus C, \\ Denmark \\ esa@inano.au.dk
}

\begin{abstract}
Look around you. The diversity and complexity of life on earth is overwhelming and data continues to grow. In our desire to understand and explain everything scientifically from molecular evolution to supernovas we depend on visual representations. This paper investigates visual representations of the bacteria Streptococcus pneumoniae by use of ink, watercolours and computer graphics. We propose a novel artistic visual rendering of Streptococcus pneumoniae and ask what the value of these kind of representations are compared to traditional scientific data. We ask if drawings and computer-assisted representations can add to our scientific knowledge about this dangerous bacterium. Is there still a role for the scientific illustrator in the scientific process and synthesis of scientific knowledge?
\end{abstract}

Bacteria. Streptococcus pneumonia. Visual representation. Molecular landscape. Data visualisation. Drawing, watercolour. Computer graphics.

\section{INTRODUCTION}

Representing data is not a neutral and unbiased practice. The visual result is usually a highly subjective composition. We use drawings and refined sketches as an active tool in the process of understanding and simplify the natural elements outlining our world. The visual, often artistic and aesthetic, approach is essential to a creative and advanced process producing groundbreaking scientific results.

How we think about and publish biological data has changed profoundly during the last couple of centuries. For example, consider the Origin of Species by Charles Darwin, having only one figure of a phylogenetic tree when published in 1859, and James D. Watson and Francis Cricks' article of the structure of DNA having only one simple hand drawing of the double helix in 1968. By comparison the Human Genome published in Nature in 2001 had 49 figures accompanying the article (Lander et al. 2001). Visual representations are becoming increasingly important to our scientific interpretation of data.

We propose a novel artistic visual rendering of Streptococcus pneumoniae (Figure 1) inspired by previous work done by David Goodsell (Goodsell
2009) and ask what the value of these kinds of representations are. Is there still a role for the scientific illustrator?

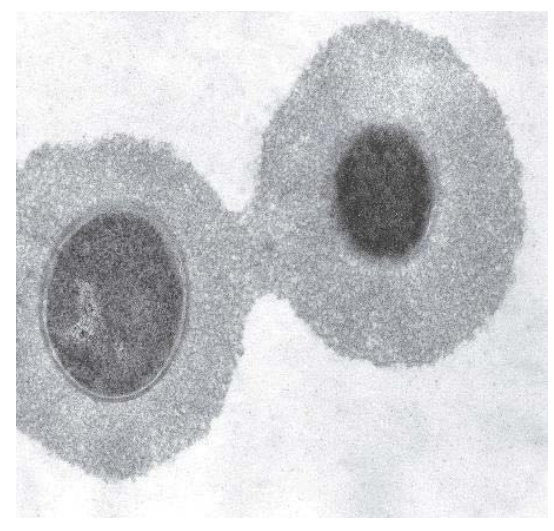

Figure 1: Electron microscopy image of the Streptococcus pneumoniae capsule, cell wall and plasma membrane (Reproduced with permission from Professor Uffe B. Skov Sørensen)

\section{VISUAL CHALLENGES}

For molecular and nanoscale data we are faced with a wide range of decisions and challenges where level of accuracy, scale, shape, colour, design, dynamics and comprehensibility need to be weighed. These decisions of information and data 
design are often made unconsciously, and routine visualisations are predominantly composed from standard software packages developed by computer scientists. We explore what, if anything, drawings, watercolours and computer-assisted representations will add to our scientific knowledge of Streptococcus pneumoniae.

The nanoscale world of molecules is invisible to the naked eye. In fact, molecules are smaller than the wavelength of light, which means that we can't see them with a light microscope. To address this problem scientists use indirect methods such as $\mathrm{x}$ ray crystallography, nuclear magnetic resonance spectroscopy, electron microscopy, or atomic force microscopy to observe the atomic structure of molecules.

The molecular world uncovered by scientists is often portrayed as colourful, twisted and intertwined molecular structures floating in space, having no clear relationship to other cellular components. In most cases the colour scheme for these structures is chosen arbitrarily with no scientific purpose or relation. The truth is, that our molecular interior is not a spectacle of beautiful multi-coloured structures.

Any molecule in a typical bacterial cell travels through the molecular world in random motion and with great speed, bumping into multiple molecules every second. The static image that we propose gives only a single glimpse of this crowded and dynamic molecular world.

Even though the artistic rendering poses some scientific and creative challenges it enables us to bring together information from many sources and display them in one illustration. It makes it easier to conceive and visualize the biological molecules in their proper context of a crowded living cell than, for example, an electron micrograph does. It also gives sense of size and distances in molecular landscapes and provokes scientific questions.

\section{STREPTOCOCCUS PNEUMONIAE}

Streptococcus pneumoniae is one of the four most frequent microbial killers worldwide (Figure 1). It causes a wide range of serious diseases such as meningitis, septicaemia and pneumonia, and less serious ones such as otitis media, sinusitis and bronchitis (Bek-Thomsen 2012, Sørensen 1988). It's found in the human upper respiratory system and the human nasopharynx is the only natural setting for it.

According to the World Health Organization the most common severe infection caused by the bacteria is pneumonia, accounting for more than
$95 \%$ of all pneumococcal diseases globally. In 2000, globally, pneumococcal infections caused about 14.5 million cases of severe disease and more than 820,000 deaths in children below the age of five, with the majority of deaths occurring in developing countries (World Health Organization 2012).

We have chosen Streptococcus pneumoniae based on its complex biological structure, widespread prevalence, and to some extent overlooked seriousness. There is, to the best of our knowledge, no comparable visual interpretation in the scientific literature yet.

\section{SCIENTIFIC NARRATIVES: TELLING STORIES FROM REAL LIFE}

We follow the artistic and narrative style of artist and molecular scientist David Goodsell in designing the molecular architecture and exploring the visual narratives of the complex molecular processes inside Streptococcus pneumoniae. Far from being a typical textbook diagram, David Goodsell's images display organized activity presenting many of the processes in cells (Figure 2). We will apply the same narrative design by explaining the structural elements of Streptococcus pneumoniae such as proteins, nucleic acids, polysaccharides, and lipid membranes in terms of molecular interactions and processes. Since this has never been done before, we hope to stimulate new scientific thoughts about the inner life of this particular bacterium.

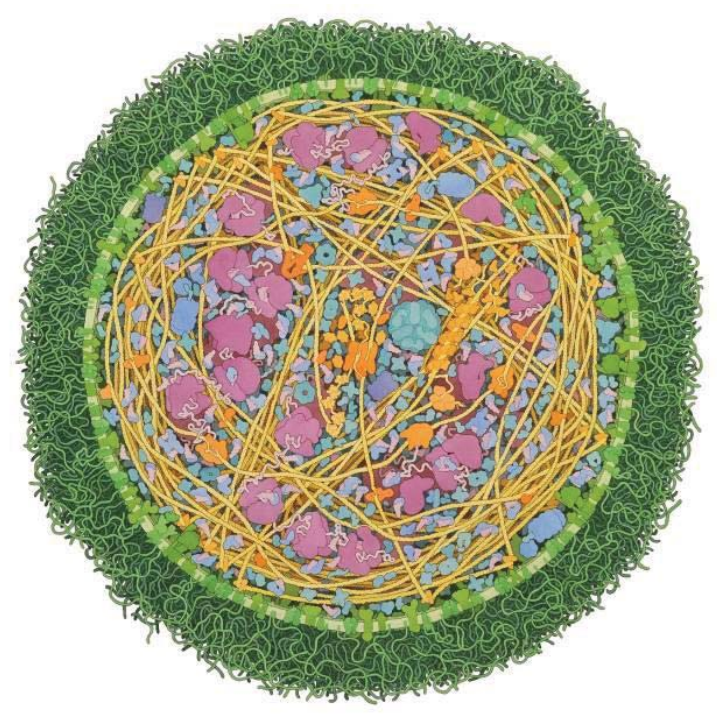

Figure 2: A Mycoplasma mycoides cell (Illustration by David S. Goodsell, the Scripps Research Institute, 2011)

To illustrate the storytelling potential of our visualisation of Streptococcus pneumoniae we present two examples of typical molecular narratives. The two following examples are adopted 
from and based on David Goodsell's image of the mycoplasma mycoides cell.

\subsection{Protein synthesis}

Protein synthesis is the process where cells create new proteins. A detail from figure 2 illustrates the process of building new proteins (Figure 3 ). The genetic code is stored in DNA (A). So-called topoisomerase (B) assists as RNA polymerase (C) loosens the DNA and builds a messenger RNA (D). Ribosomes (E), large and complex molecular machines, link amino acids together in the order specified by messenger RNA molecules and transfer RNA (F).

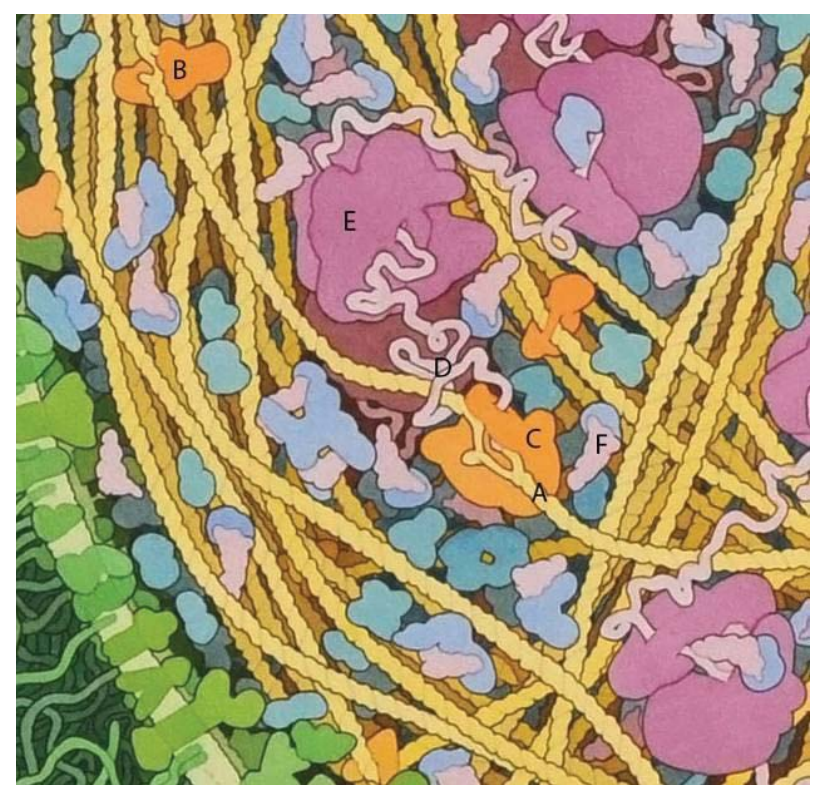

Figure 3: Detail of Figure 2, showing some of the molecules involved in protein synthesis in a bacterial cell

\subsection{Producing energy}

Another example of a narrative vital to the living bacteria is the production of energy in the cell. Another detail from Figure 3 highlights some of the molecules involved in producing energy (Figure 4). Glycolytic enzymes (A) break down sugar and produce ATP (adenosine triphosphate), the energy source of cells. The ATP synthase (B) consists of an enzyme, a molecular motor, an ion pump, and another molecular motor, all of which constitutes a nanoscale machine producing energy.

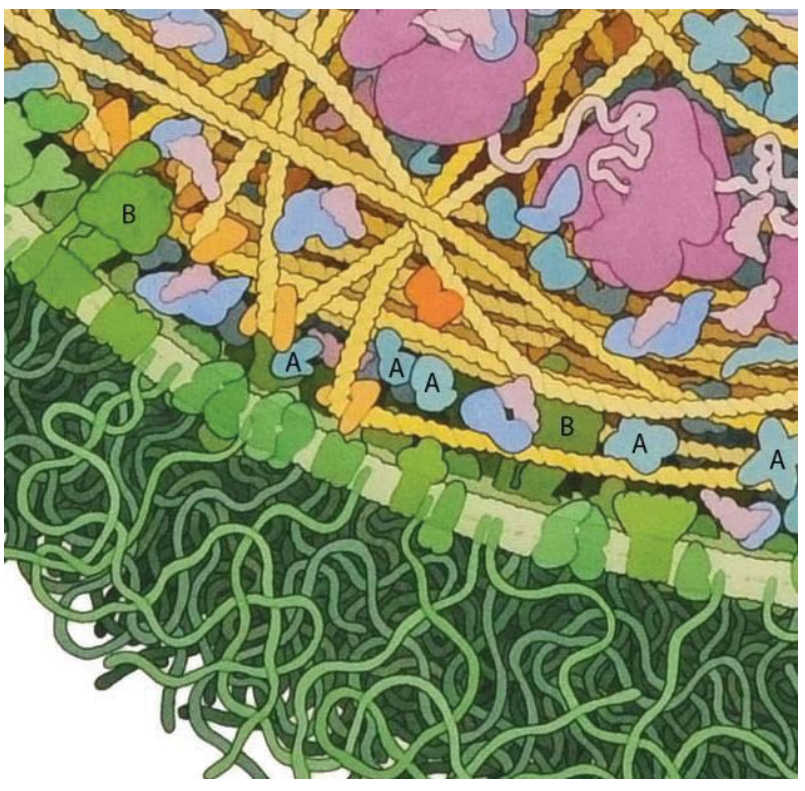

Figure 4: Detail of Figure 2, indicating some of the molecules involved in powering the cell

\section{COLLECTING THE DATA: ASSEMBLING THE STRUCTURE}

Taking advantage of previous artistic renderings of cell environments (Goodsell 2009, Pollard et al. 2008) and resources such as the RCSB Protein Data Bank (www.rcsb.org), we will produce visual representations of all necessary molecules and cellular elements present in Streptococcus pneumoniae. We will include the proper number of molecules, in the right places and having the scientific accurate proportions.

\subsection{Scientific resources}

We use a long list of scientific data and resources describing complex individual molecules and molecular processes. To get an overview of the complete cell structure and to determine the structure of the cell wall in particular, we investigate micrographs and image data from electron microscopes. We use data from x-ray crystallography, nuclear magnetic resonance spectroscopy, and atomic force microscopy to establish the atomic structures of the individual molecules. Most molecular structures needed are found via the RCSB Protein Data Bank (www.rcsb.org) (Table 1).

The data found in the RCSB Protein Data Bank shows structures located inside the cell, components embedded in the plasma membrane, as well as the sugary armour protecting the bacteria. We use micrographs and structural data to determine morphology and location of the different molecular elements. 
Table 1: A short list of examples of visual representations from different scientific sources. The first column names the molecule; the second column lists the entries in the RCSB Protein Data Bank (www.rcsb.org); the third column shows the structures drawn by computer graphics adopted from RCSB PDB-101, and the fourth column shows sketches of the structures as these will appear in our drawing. We use a simplified structure in the final visualisation of Streptococcus pneumoniae. The structures are represented in grey scale to avoid visual distraction.

\begin{tabular}{|c|c|c|c|}
\hline \multirow[t]{2}{*}{ Molecule } & \multicolumn{3}{|c|}{ Scientific resource } \\
\hline & $P D B$ entries & Structure drawing & Sketches \\
\hline DNA & $1 b n a$ & Ther & \\
\hline $\begin{array}{l}\text { Ribosome, } \\
\text { small subunit }\end{array}$ & 1fka; 1fjg & & \\
\hline $\begin{array}{l}\text { Ribosome, } \\
\text { large subunit }\end{array}$ & 1 ffk & & \\
\hline $\begin{array}{l}\text { ATP } \\
\text { synthase }\end{array}$ & $\begin{array}{l}\text { 1c17; 1e79; } \\
2 a 7 u ; 1 / 2 p\end{array}$ & & \\
\hline
\end{tabular}

\section{MAKING THE MODEL}

We focus on two different forms of visual representation:

(i) Drawings with watercolour paint magnifying objects one million times (consistent to scale allowing for comparison with previous work composed by David Goodsell).

(ii) Computer-generated images showing atomic details of individual molecules.

Our scale of the entire bacteria $(0.8-1 \mu \mathrm{m}$, even without the pili) makes it difficult to draw every molecule, showing each atom represented by a sphere. Instead, we use a simplified space-filling representation adopted from David Goodsell, catching the overall shape of each molecule without necessarily showing the location of every atom (Table 1).

\subsection{Molecular architecture of the cell wall}

Typically, Streptococcus pneumoniae is found as two cells sharing parts of their cell wall. The cells are enclosed completely by a polysaccharide capsule. So far there are found 95 distinctive variations of Streptococcus pneumoniae, characterized by their different polysaccharide capsule. By use of immune-electron microscopy (Sørensen 1988), tunnelling electron microscopy and scanning transmission electron microscopy (Hilleringmann 2009), studies have determined the uniform distribution of the polysaccharides on the cell wall (sugar chains), and the molecular architecture of the pili. Pili are very long hair-like protein structures. The pilus is crucial for pathogenic bacteria needing to attach to host cells and establish an infection. They are approximately $6 \mathrm{~nm}$ wide and more than $1 \mu \mathrm{m}$ long (Figure 1). The pili are composed of a single string of so-called RrgB monomers and have a polarity defined by nose-like bumps (Hilleringmann 2009).

\subsection{Visual concepts}

A key challenge is to design an environment based on actual raw data rather than approximations, and to be able to handle great differences in spatial scale. In research settings, the relationship between a single molecule and other molecules, and its effects on an entire organism, is often implicit. 


\section{FUTURE PERSPECTIVES}

The detailed map of Streptococcus pneumoniae that we propose to construct will provide a visual encyclopaedia designed to generate an understanding of the complex functions of the bacteria. By collecting and presenting data in this way we might expose evidence challenging the scientific knowledge.

The combination of hand-drawings in ink and watercolour, together with computer graphics illustrations enable us to bring together information from many sources and display them in one illustration.

In the process we will create elements for making new and even larger molecular landscapes that might inspire scientists working with nanomedicine to develop therapeutic strategies for treating infectious diseases.

\section{ACKNOWLEDGEMENTS}

We are grateful to Professor Mogens Killian and Professor Uffe B. Skov Sørensen for electron micrograph material and detailed discussions on the biology of Streptococcus pneumoniae.

\section{REFERENCES}

Bek-Thomsen, M. (2012) Genetic Diversity and Relationships of Streptococcus pneumonia and its Close Commensal relatives. Aarhus University, Aarhus. PhD Dissertation.

Goodsell, D. S. (2009) The Machinery of Life. Springer, New York, USA. $2^{\text {nd }}$ edition.

Hilleringmann, M., et al. (2009) Molecular architecture of Streptococcus pneumoniae TIGR4 pili. The EMBO Journal, 28, 3921-3930.

Lander, E. S., et al. (2001) Initial sequencing and analysis of the human genome. Nature, 409, 860921.

Pollard, T. D., et al. (2008) Cell Biology. Saunders Elsevier, Philadelphia.

Sørensen, U. B. S., et al. (1988) Ultrastructural location of capsules, cell wall polysaccharide, cell wall proteins, and $\mathrm{F}$ antigen in pneumococci. Infection and Immunity, 56(8), 1890-1896.

World Health Organization (2012) Measuring impact of Streptococcus pneumoniae and Heemophilus influenzae type $b$ conjugate vaccination. http://www.who.int/vaccinesdocuments/ (retrieved March 10, 2014). 